\title{
Galactic Abundances: Report of Working Group 3
}

\author{
B. Klecker*, V. Bothmer ${ }^{\dagger}$, A. C. Cummings**, J. S. George**, J.W. Keller ${ }^{\ddagger}$, E. \\ Salerno $^{\S}$, U. J. Sofia", E. C. Stone**, F.-K. Thielemann", M. E. Wiedenbeck ${ }^{\dagger \dagger}$, F. \\ Buclin $^{\S}$, E. R. Christian ${ }^{\ddagger}$, E. O. Flückiger ${ }^{\S}$, M. Y. Hofer ${ }^{\S \S}$, F. C. Jones ${ }^{\ddagger}$, D. \\ Kirilova $^{\llbracket \uparrow}$, H. Kunow*** ${ }^{* *}$ M. Laming ${ }^{\dagger \dagger}$, C. Tranquille ${ }^{\S \S}$ and K.-P. Wenzel ${ }^{\S \S}$ \\ ${ }^{*}$ Max-Planck-Institut für extraterrestrische Physik, 85740 Garching, Germany \\ ${ }^{\dagger}$ Max-Planck-Institut für Aeronomie, 37191, Katlenburg-Lindau, Germany \\ ${ }^{* *}$ Caltech, Pasadena, CA 91125, USA \\ ${ }^{\ddagger}$ Laboratory for Extraterrestrial Physics, GSFC, Greenbelt, MD 20 771, USA \\ ${ }^{\S}$ Physikalisches Institut, University Bern, CH-3012 Bern, Switze rland \\ "Department of Astronomy, Whitman College, Walla Walla, WA 9936 2, USA \\ "Dept. of Physics \& Astronomy, Univ. of Basel, Klingelbergstrasse 82, CH-4056 Basel, Switzerland \\ ${ }^{\dagger} J P L$, Caltech, Pasadena, CA 91109, USA \\ \$NASA-GSFC, Greenbelt, MD 20771, USA \\ $\S$ Space Science Department of ESA, ESTEC, 2200 AG Noordwijk, NL \\ If Institute of Astronomy, Bulgarian Academy of Science, Sofia, B ulgaria \\ ${ }^{* * *}$ IEAP, Universität Kiel, 24118 Kiel, Germany \\ ${ }^{\dagger}$ Naval Research Laboratory, Washington DC 20375, USA
}

\begin{abstract}
We summarize the various methods and their limitations and strengths to derive galactic abundances from in-situ and remote-sensing measurements, both from ground-based observation $\mathrm{s}$ and from instruments in space. Because galactic abundances evolve in time and sp ace it is important to obtain information with a variety of different methods covering different regions from the Very Local Insterstellar M edium (VLISM) to the distant galaxy, and different times throughout the evolution of the galaxy. We discuss the study of the present-day VLISM with neutral gas, pickup ions, and Anomalous Cosmic Rays, the study of the local

interstellar medium (ISM) at distances $<1.5 \mathrm{kpc}$ utilizing absorption line me asurements in H I clouds, and the study of galactic cosmic rays, sampling contemporary $(\sim 15 \mathrm{Myr}) \mathrm{s}$ ources in the local ISM within a few kiloparsec of the solar system. Solar system abundances, derived from solar abundances and meteorite studies are discussed in several other chapters of this volume. They provide samples of matter from the ISM from the time of solar system format ion, about $4.5 \mathrm{Gyr}$ ago. The evolution of galactic abundances on longer time scales is discussed in the context of nuclear synthesis in the various contributing stellar objects.
\end{abstract}

\section{INTRODUCTION}

In Working Group 3 (WG3) of the SOHO-ACE workshop the various methods for obtaining galactic abundances have been discussed. Because galactic abundances are neither constant in time nor homogenous in space, it is important to obtain information on the galactic abundances with a variety of different methods covering different regions in space from the Very Local Interstellar Medium (VLISM) to the distant galaxy, and different times from the present to the early days of the galaxy, $\sim 10^{10}$ years ago. These various methods with their different measurement techniques have different limitations and strengths. In this report we will summa- rize these techniques or refer to other papers presented at this conference whereever possible to reduce overlap, provide references to recent results, discuss the limitations and strengths of the methods and give an outlook to future plans or further measurements needed.

The composition of the present-day VLISM can be studied by analyzing samples of the neutral interstellar gas that penetrates deep into the heliosphere. With presently available instrumentation it is possible to study this sample at various stages, starting with the neutral gas itself. A summary on neutral interstellar gas measurements available at present and in the near future is provided in chapter 1.

A more evolved sample of the VLISM are interstellar 
pickup ions created by ionization by solar UV or charge exchange with solar wind protons. In the pickup process the ions are accelerated up to twice the solar wind velocity, i.e. to energies up to several $\mathrm{keV} /$ nucleon and can then be measured with ion composition mass spectrometers. For a summary of compositional measurements of the VLISM derived from the observation of pickup $i$ ons see Gloeckler and Geiss, this volume [1].

Another, even more processed, sample of the VLISM are the Anomalous Cosmic Rays (ACR). In the 25 years since their discovery in low-energy quiet-time helium, nitrogen, and oxygen spectra, it has been well established that the origin of ACRs are pickup ions that are further accelerated in the outer heliosphere to energies of hundreds of $\mathrm{MeV}$ [2], presumably at the heliospheric termin ation shock [3], which is currently thought to be located at about $90 \mathrm{AU}$ fro $m$ the Sun. A summary of ACR measurements is provided in chapter 2 on Anomalous Cosmic Rays.

With relative speeds of interstellar neutrals of $\sim 25$ $\mathrm{km} / \mathrm{s}$ and acceleration times of ACRs of a few years, the time delay between entering the heliosphere as neutral gas and the observation in the inner heliosphere as neutrals, pickup ions or ACRs is only of the order of a few years. Thus, all three samples provide information on the present-day abundances of the VLISM.

The present-day interstellar medium (ISM) at larger distances can be probed with

absorption line studies. H I clouds can provide compositional information on the present ISM at distances to about 1500 pc for a limited number of elements. These isotopic abundances in meteorites see Hoppe, this volume [4], and $r$ eferences therein.

Galactic abundances are neither constant in time nor are they universal on a sol ar level. Their evolution in space and time reflects the history of star formation and the lifetimes of the various contributing stellar objects (see [5], and references the rein). Thus, the evolution of galactic abundances can be investigated by the study of the surface composition of other stars of different age, i.e. different metallicity. Chapter 5 on Stella $r$ Abundances provides a summary on stellar abundances as indicators of galactic evolution.

\section{NEUTRALS}

The solar system is moving through the VLISM approximately into the direction of Scorpio with a speed of about $25 \mathrm{~km} / \mathrm{s}$. As a result of this motion, an interstellar wind of neutral particles (mainly hydrogen with approximately 10 percent of helium) crosses the heliopause and enters the heliosphere with the same velocity. Neutrals from the VLISM and high energy GCRs with energies exceeding $\sim 1 \mathrm{GeV}$ are the only particles that can go beyond the heliopause. The solar wind plasma, being highly magnetized compared to the interstellar medium, acts as a shield for the low-energy ionized VLISM component.

The interstellar flux after entering the heliosphere is focused by the sun's gravity to form a focusing cone in a direction pointing away from the incoming local interstellar wind. The notable exception is the hydrogen

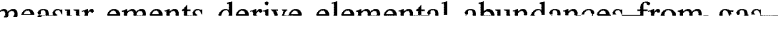




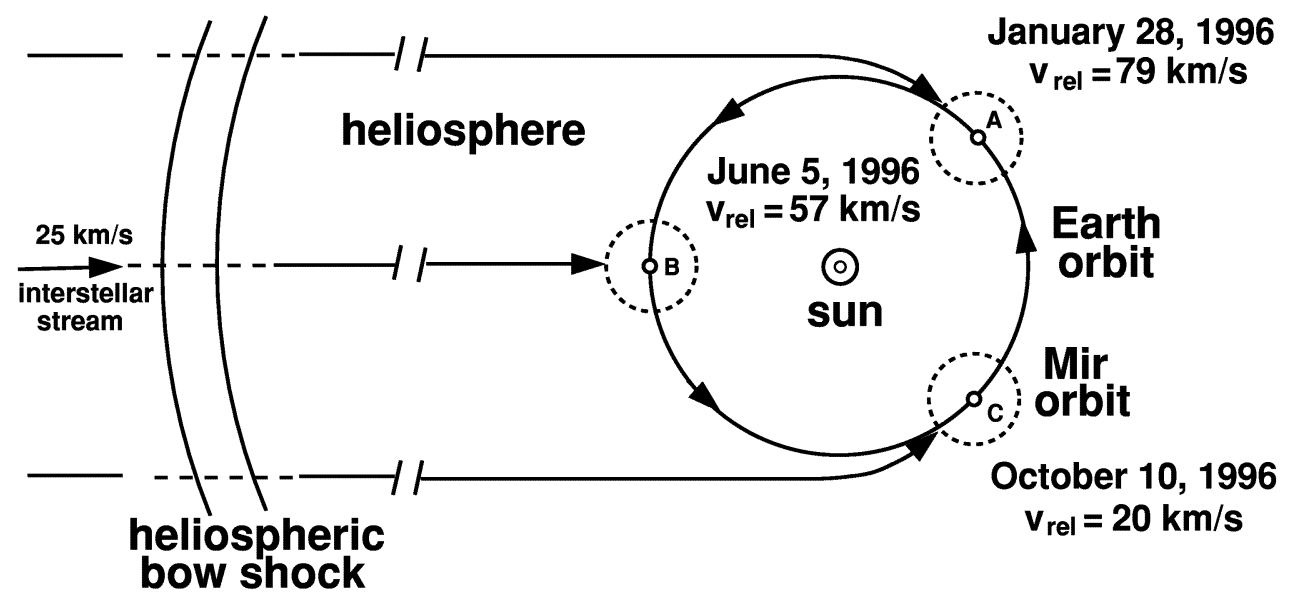

FIGURE 1. Seasonal variation of speed and direction of the interstellar neutral flux as consequence of the revolution of the Earth around the Sun. In December, the Earth is passing through the most dense part of the neutral gas, the focusing cone in the "downwind" direction (not shown in the picture), whil e from January to May, when the Earth is "upwind" of this pile-up, the helium flux density is lower but its velocity relative to Earth is higher.

elemental and isotopic composition. A complete review of the techniques for the detection of energetic neutral atoms currently in use and those developed for future applications in space research can be found in [6]. Working Group 3 discussed some of these techniques focusing the attention mainly on the pinhole camera principle used for the GAS experiment on Ulysses and the foil collection technique used for the COLLISA experiment on the Russian Space Station Mir whose recent results have been presented at this conference.

\section{Pinhole camera: the GAS experiment on Ulysses}

The GAS experiment on board the Ulysses spacecraft used, for the first time, an in situ technique to measure the properties of the local interstellar neutral gas. Up to now, the experiment allowed the determination of density, bulk velocity relative to the solar system and temperature of the interstellar flow by measuring the neutral helium penetrating the heliosphere [7]. In principle the GAS instrument (described in detail by Witte et al. [8]), detecting secondary particles emitted by a conversion plate when this is hit by the helium atoms, acts as a pinhole camera. Since the instrument is not equipped with any imaging detector, it is necessary to perform a two-dimensional scan over the hole sky in order to obtain a global picture of the neutral helium flux [9]. The efficiency of the instrument is highly energy dependent and neutral helium can only be detected when the particle's energy is larger than $\sim 30 \mathrm{eV}$. Thus the detection of interstellar gas is only possible when the velocity vectors of the interstellar flux and Ulysses add up to a relative ve- locity high enough for detection. The energy-dependent efficiency also makes the sensor practically blind for the interstellar hydrogen. The detection probability for heavier elements would be high enough but the fluxes are so small that one can easily assume to measure exclusively interstellar neutral helium.

The advantages of the Ulysses GAS instrument are the rather simple design and the simple operation in space. Besides, the detection efficiency for energetic helium atoms is high (probably there is no other technique with comparable detection efficiency for helium). On the other hand, no mass and/or energy analysis of the detected particles can be achieved with this technique as well as no extraction of meaningful data is possible if the strict geometric and energetic instrumental requirements are not fulfilled.

So far, the GAS experiment onboard Ulysses has been the only instrument based o $\mathrm{n}$ the pinhole camera principle that has been used to study the neutral component of the interstellar inflow. However, future missions aiming to investigate the interstellar gas will very likely use an instrument of the Ulysses GAS type, probably extended to a full pinhole camera with a position-sensitive detector (alleviating, in this way, the need for a scan platform and for a spinning spacecraft).

\section{The foil collection technique: the COLLISA experiment on Mir}

The foil collection technique represents one of the earliest methods for the study of the elemental and isotopic composition of noble elements in space gas flows. The 
method is based on thin metal foils that are directly exposed in space to the stream of particles whose composition needs to be studied. If the kinetic energy is sufficiently large (usually $\geq 50 \mathrm{eV}$ ), the particles ramming against the surface of the foil get trapped in its atomic structure and remain there until a change in the foil's pressure or temperature causes their release. The choice of thin foils as trapping medium results from their extremely easy handling in space. Besides, while the small thickness assures a very low contamination from particles initially contained in the medium, compared to more massive objects, the possible use of large surfaces allows the capture of large amounts of particles. Once the foils have been exposed for a period of time long enough to allow a considerable capture of particles (from days to years, according to the particle flux density and to the experimental setup), they are recovered from space and delivered to Earth. Here, with a special procedure, the particles are extracted from the foil and sent to a noble gas mass spectrometer where their abundances can be measured. Due to the very low presence of noble gases both in the foils and in the laboratory mass spectrometers, the foil collection technique represents one of the most convenient methods for the analysis of this kind of particles. Other advantages offered by this method are the simple instrumentation and operation needed in space, plus the fact that the mass spectrometric analysis can be performed on Earth with resolutions and sensitivities much higher than is possible on a space-born instrument.

In 1965 Signer et al. [10] proposed for the first time an experiment aim ing to determine the elemental and isotopic composition of the solar wind, by means of the exposure of appropriate foils to the interplanetary plasma and subsequent extraction and analysis of the particles trapped in the foils. Such an experiment was later on carried out during the Apollo 11 and 12 missions, when aluminum foils (mounted on a pole stuck in the lunar soil) were exposed to flux of solar wind particles from the surface of the Moon [11]. In 1972 the foil sampling technique was used again to measure the noble gas fluxes in the auroral primary radiation $[12,13]$. This time aluminum and platinum foils were mounted on two Nike-Tomahawk rockets that were launched directly into bright auroras. Some years later, during the Skylab missions, some foils were exposed to collect precipitating magnetospheric isotopes [14]. The foil collection technique was utilized for studying the neutral atoms coming from the Local Interstellar Cloud (LIC) for the first time in 1991 in the framework of the Interstellar Gas Experiment (IGE). On that occasion, several beryllium-copper foils were exposed to the flux of neutral interstellar particles from the outside of the Long Duration Exposure Facility (LDEF) satellite $[15,16]$. Unfortunately, a malfunctions of the IGE electrical system during flight did not allow the exposure of all the foils, while the few foils exposed resulted highly contaminated with energetic magnetospheric particles.

Recently, for the COLLISA experiment, this method has been used to collect a sample of interstellar neutral matter with the aim to determine the helium and neon isotopic ratios in the LISM. The experiment was performed on board the Russian space station Mir. During Spring 1996 four beryllium-copper foils were exposed for $\sim 60$ hours to the flux of the interstellar neutrals. As already mentioned, in this period of the year the particle collection is particularly effective since the Earth moves in the upwind direction of the interstellar flux of neutrals, reaching the maximum relative velocity $(\sim 60-80$ $\mathrm{km} / \mathrm{sec}$ ). Such a condition enhances the particles' kinetic energy up to $\sim 25 \mathrm{eV} /$ nucleon. increasing the efficiency by which they get trapped into the foils. After exposure in space, the foils were recovered by the cosmonauts and brought back to Earth by the U.S. space shuttle Atlantis. The mass spectrometric analysis of the foils exposed to the interstellar flux has resulted in the clear detection of large amounts of ${ }^{3} \mathrm{He}$ and ${ }^{4} \mathrm{He}\left(\sim 10^{6}\right.$ and $\sim 10^{10}$ atoms respectively) with isotopic ratio ${ }^{3} \mathrm{He} /{ }^{4} \mathrm{He}$ equal to $\left\{1.71_{-0.42}^{+0.50}\right\} \times 10^{-4}[17]$.

\section{New approaches for the study of the VLISM neutral component}

Measurements of neutral atom density and composition require highly sensitive techniques because their fluxes in space are low. Detection and analysis of neutral atoms often require their ionization and this should be accomplished with efficiencies that approach unity. Long integration times are necessary for rare elements and large-aperture instruments should be considered when possible. Sample return techniques as discussed above can provide the necessary sensitivity but are not always applicable since low FIP elements from the VLISM do not arrive at $1 \mathrm{AU}$ where sample return is most easily accomplished. Also, while the foil technique has been demonstrated to work well for the noble gases, it remains to be seen if the results for other elements will be of comparable quality. For in situ measurements new approaches and developments are required (see Wurz [6] for a review). There is reasonable hope, for example, that a measurement of the interstellar gas will be also possible with the IMAGE/LENA instrument. Besides, measurements of the interstellar neutral hydrogen distribution performed by the SWAN instrument on SOHO through observations of the scattered Lyman alpha (UV) radiation are already available [18].

At the conference, Keller et al. [19] have proposed neutral atom ionizat ion through charge exchange collisions with reactive metal vapors. These low ionization 
potential gases are mechanically confined to a collision cell through the spinning of rotor blades at turbomolecular speeds. Neutral atoms with finite electron affinity can pass through the cell and emerge as negative ions. The collisional cross section for charge exchange are large so that a relatively low density of vapor $\left(\sim 10^{13} \mathrm{~cm}^{-3}\right)$ is required for good ionization efficiency. The cell is opaque to the thermal metal vapor inside but partially transparent to the fast moving atoms that are to be detected. The product of the path length through the cell, the vapor density, and the collisional ionization cross section determines the ionization probability of an atom that passes through ([19]).

\section{ANOMALOUS COSMIC RAYS}

Anomalous cosmic rays (ACRs) are an accelerated sample of the local interstellar medium $[2,3]$. They begin as interstellar neutral gas which drifts into the heliosphere at a speed of about $25 \mathrm{~km} \mathrm{~s}^{-1}$. A portion of them become ionized by the processes of photoionization and/or charge exchange with the solar wind. These newly created ions are immediately picked up by the interplanetary magnetic field which is embedded in the expanding solar wind. The ions are then further accelerated in the outer heliosphere, gaining most of their energy at the solar wind termination shock, which is currently thought to be located at about $90 \mathrm{AU}$ from the Sun [20].

\section{Experimental Techniques}

ACRs are observed in the multi-MeV/nucleon energy range principally by cosmic-ray solid-state detector telescopes on a variety of spacecraft in the heliosphere, including Voyager, ACE, SAMPEX, Wind, and Ulysses (see review in [21]). The most comprehensive and least fractionated sample of elemental abundances are obtained from the Voyager spacecraft in the outer heliosphere. Charge-state measurements are carried out at 1 $\mathrm{AU}$ by instruments on the SAMPEX spacecraft [22] in polar orbit around the Earth using the geomagnetic filtering technique [30]. SAMPEX also samples ACRs that have become trapped in the Earth's magnetosphere. The absolute intensity of this trapped population is $>100$ times that in the interplanetary medium [23]. Isotopic composition results for some elements are available from several spacecraft, including Voyager, SAMPEX, and ACE. The latter offers the statistically most accurate and comprehensive set of results.

\section{Limitations and advantages of the method}

In order to derive the composition of the local interstellar medium, account has to be taken of two additional fractionation steps beyond those affecting pickup ions. The two steps are the 1) injection and acceleration of the pickup ions at the termination shock and 2) the propagation inwards to the point of observation from the solar wind termination shock. By comparing observations from the two methods, it is possible to determine the nature of these fractionation steps.

ACRs offer a direct measurement of the isoptopic composition of the present-day VLISM [28]. For neutrals and pickup ions this is so far possible only for ${ }^{3} \mathrm{He}$ $[17,25]$. In addition, the ACRs are critical in estimating the relative injection/acceleration efficiency at the termination shock. The pickup ion observations yield the flux of ions flowing into the shock and the ACR observations yield estimates of the resulting accelerated energy spectra. Comparing the two sets of observations yields the injection/acceleration efficiencies. These efficiencies can be determined for $\mathrm{H}, \mathrm{He}, \mathrm{N}, \mathrm{O}$, and $\mathrm{Ne}$. In the case of $\mathrm{Na}$ and Ar, which are measured in the ACRs but not in the pickup ions, it is possible to estimate their interstellar neutral densities by extrapolating the injection/acceleration efficiency from that determined for $\mathrm{N}$, $\mathrm{O}$, and $\mathrm{Ne}$. Another strength is that the charge state of the ACRs as a function of energy can be measured using the geomagnetic filtering technique with a polar orbiting satellite such as SAMPEX.

\section{Results}

- The elemental composition of $\mathrm{H}, \mathrm{He}, \mathrm{N}, \mathrm{O}, \mathrm{Ne}, \mathrm{Na}$, and Ar in the neutral VLISM, as deduced from ACR and pickup ion measurements, appears to be in good agreement with expectations from a recent model of the ionization state of the local interstellar medium $[24,26]$.

- Spectral features at low energy in other elements are seen which appear to require a source other than the VLISM [24].

- The isotopic composition of ACR O and $\mathrm{Ne}$, and therefore the present-day VLISM, is consistent with that of the solar system within reasonably large uncertainties [27, 28, 29].

- Anomalous cosmic rays are primarily singly charged below about $350 \mathrm{MeV}$ with a transition to higher charge states above that value $[30,31,32]$.

- Heavier ions are preferentially injected into the ACR acceleration mechanism [1, 24, 33]. 


\section{Outstanding problems and questions}

- What are the limits on galactic evolution and what are the implications?

- What is the injection process at the solar wind termination shock?

- What is the origin of the minor ACR ions, e.g., $\mathrm{Mg}$, $\mathrm{Si}$, and $\mathrm{S}$ (see e.g. [21], and references therein)?

\section{The Future}

Greatly improved statistical accuracy and reduction of background due to galactic cosmic rays could be accomplished by an instrument with large collecting power in a polar orbit around Earth.

Measurements of the source spectra of ACRs would provide better estimates of the elemental and isotopic composition of the local interstellar medium, as well as better information on the injection and acceleration process. Voyager should provide exploratory measurements in the near future but a more advanced instrument on a mission to the termination shock is desired.

Because of the limitations due to the fractionation steps dicussed above, progress in 1) modeling the heliosheath region with respect to charge-exchange filtration effects on both the neutral and ionized component of the VLISM, and 2) modeling of the transport, preacceleration, injection, and acceleration of pickup ions to become ACRs will improve the derivation of the LISM composition from ACR measurements.

\section{H I CLOUDS}

H I clouds can provide reliable Galactic abundances for several elements. In fact, interstellar studies have used the gas-phase measurements of neutral interstellar clouds together with grain models to determine a local Galactic composition for $\mathrm{N}, \mathrm{O}$ and $\mathrm{Kr}[34,35,36,37,38,39]$. These are the few elements for which we have gas-phase measurements and a good understanding of their dust abundances. Useful limits can be placed on the Galactic abundances of some species with less-well understood dust-phase characteristics, e.g. C and Sn. Although this method is quite limited in the elements that it can be used for, some very important species are among them.

\section{Method}

The method of determining the Galactic abundance from neutral (H I) clouds has an observational and a theoretical component. The ISM is composed of gas (the abundance of which can be measured) and dust (the abundance of which can be determined through grain modeling). If we add these two components together for a given element, we can easily obtain its Galactic abundance.

The gas-phase abundances are determined through absorption line studies. Most resonance lines of the dominant ions in neutral interstellar clouds occur in the ultraviolet region of the spectrum. Therefore one needs a high resolution UV spectrograph to make the measurements. The GHRS and STIS instruments aboard the Hubble Space Telescope (HST) are well designed for this purpose. The most reliable gas-phase abundances come from weak transitions of species. For extremely weak lines, the equivalent width or line strength alone gives an accurate abundance if the oscillator strength of the transition is well determined. These atomic constants have been updated and improved greatly since the HST was launched. This was necessitated by the high quality of the spectroscopic data. For absorption lines that are stronger than the weak limit, corrections must be made for unresolved saturation in the line cores. This is a straightforward procedure that is independent of the component structure of the absorbing regions $[40,41,42]$. Therefore, abundances in the gas can be well-determined without invoking any model dependent parameters. Gas-phase abundances determined in this way are usually acurate to 0.05 dex or $\sim 10 \%$. Abundances from stronger absorption lines are much less reliable and therefore less useful for Galactic abundance studies.

The dust-phase abundances of the elements are necessarily model dependent because they cannot be directly observed. All of the cosmically abundant elements have some limits on their dust incorporation which are set by theories trying to reproduce extinction curves. However, theses grain models are not unique, so the limits placed on an elemental dust incorporation often has a large range. Only the elements which show the same levels of dust incorporation in all (or at least most) of the theories can be trusted for their dust-abundances. These elements, most of which are completely absent from dust [43], are the ones for which we can determine Galactic abundances.

\section{Samples}

The interstellar cloud samples for these studies are diffuse, $\mathrm{A}_{V} \leq 1.0$ magnitudes, $\mathrm{H}$ I regions between the sun and $\mathrm{O}$ or $\mathrm{B}$ stars. The requirement of an early-type star results from the need for enough flux in the UV (above the Lyman limit) to observe very weak lines. These stars often have large values of $v \sin i$ which allow the stellar and interstellar absorption to be disentangled. The choice 
of diffuse neutral clouds facilitates the gas-phase abundance determinations because there is usually a well defined dominant ion for each element in these regions. Also, depletion onto dust is well understood in these clouds $[44,45,34,46]$ partly because no ice mantles can exist in them. Interstellar regions observed for these studies are all relatively local; the best current samples extend to only about $1500 \mathrm{pc}$ from the sun $[47,48,49,50,51]$. This limit is imposed by current instrumentation and the flux requirements for high quality spectra. The sightlines do, however, sample a wide range of physical conditions present in diffuse clouds. This is judged by the fact that they have very different fractions of their hydrogen nuclei in the form of $\mathrm{H}_{2}$ (as opposed to $\mathrm{H} \mathrm{I}$ ), $\mathrm{f}\left(\mathrm{H}_{2}\right)=2 N\left(\mathrm{H}_{2}\right) /\left[2 N\left(\mathrm{H}_{2}\right)+N(\mathrm{H} \mathrm{I})\right]$. These values, which range from about $10^{-6}$ to 0.6 in diffuse clouds, measure the balance between formation of $\mathrm{H}_{2}$ molecules on grain surfaces and their photo- and/or chemical-destruction.

\section{Limitations and advantages of the method}

The neutral cloud method is limited in several ways. The most stringent of these is the need to understand the abundances of the elements in dust. Very few of these are known well enough for this method to work. A second limit is caused by the possibility of ionization in the gas. We are usually able to measure the dominant ion in the neutral clouds. If a substantial amount of an element is in a different ion state than expected, then we may underestimate its Galactic abundance. Previous studies have shown that this is not a problem for the species and regions that have so far been studied. Finally, unlike other methods, observations of interstellar lines reveal very little about isotope ratios because of limitations in data quality. The exception is boron for which isotope ratios have been determined from HST data [52, 53].

The greatest advantage of determining Galactic abundances from neutral interstellar clouds is that the method is so straight-forward. There is little-to-no model dependencies built into these abundances, especially if one is conservative about choosing elements with well determined dust-phase abundances. Therefore, the results from this method are extremely robust and reliable.

\section{Results from neutral clouds}

A discussion of the results based on this method are given in a paper by Sofia in these proceedings [43]. A summary of those results are given here. One of the most important findings from the interstellar studies is that the ISM within about $1500 \mathrm{pc}$ of the sun in random directions is extremely uniform in composition. This indicates that a true local Galactic abundance does exist. The uniformity of the ISM measurements contrasts young star abundances which have significantly more scatter [39].

As far as specific ISM abundances are concerned, the local abundances of $\mathrm{O}$ [50], $\mathrm{N}$ [54] and probably $\mathrm{C}$ $[47,48]$ agree well with the solar photospheric measurements of Holweger [55]. The abundance of $\mathrm{O}$ in dust is relatively well known because of mineralogy, so its Galactic abundance can be well determined. The same is true for $\mathrm{N}$ which is probably not incorporated into dust at all. Carbon's abundance in dust is not well determined. Arguments based on the $\mathrm{C} / \mathrm{O}$ ratio in young stars and the sun suggest, however, that it may be solar [39]. The fact that the local Galactic C, N and especially $\mathrm{O}$ abundances are solar is a very recently resurrected idea. Until recently the ISM community argued that the local Galactic abundance of $\mathrm{O}$ was subsolar based on gas-phase measurements of the ISM together with grain model considerations $[34,35,36,37,50]$. In the past several years, however, the reported solar abundances of $\mathrm{O}$ have been slowly dropping. The most recent photospheric abundance (reported in these proceedings [55]) is now in agreement with the abundances determined from the ISM years ago [47, 50]. This demonstrates the reliability of the neutral cloud method for determining the local Galactic composition.

Other elements in the local ISM do not appear to have solar abundances. For instance, the abundance of krypton in the ISM seems to be low compared to the solar system value [49]. Like $\mathrm{N}, \mathrm{Kr}$ is not expected to be in dust at all so its Galactic abundance should be well determined. The apparent $\mathrm{Kr}$ underabundance may simply be the result of the difficulties in measuring $\mathrm{Kr}$ in the solar system (i.e. meteorites). If this is the case, then the ISM $\mathrm{Kr} / \mathrm{H}$ value may be a good representation of the solar composition. Another element that appears not to agree with the sun is tin. Tin's incorporation into dust is not well understood so we are limited in what we can determine about its abundance. We can, however, confidently say that $\mathrm{Sn}$ is overabundant in the ISM with respect to the sun. This is because the measured gas-phase abundance alone is supersolar [51]. What we do not know, however, is to what extent it is incorporated into dust, and therefore how overabundant it is with respect to the sun.

\section{The Future}

As noted earlier, the major limit to this method is the uncertainty of the abundances of elements in dust; we can measure the gas-phase abundance for many more species than we have dust data for. Therefore, any improvements in the modeling of dust will help with the determination of Galactic abundances from the neutral 
ISM. Particularly, the dust-phase abundance of $\mathrm{C}$ would be very important since we have a very good measurement of its gas-phase abundance in the local ISM [48]. Other important species include $\mathrm{Si}, \mathrm{Mg}$ and $\mathrm{Fe}$. However these species have variable depletion levels in diffuse clouds, possibly because they are able to grow on non-ice mantles. Finding Galactic abundances for these species, therefore, requires that their elemental incorporation into both grain cores and grain mantles be well understood.

In 2003 a new spectrograph, COS, will be placed on the Hubble Space Telescope. This instrument will be able to extend ISM studies of Galactic abundances to greater distances because it will have a sensitivity that is more than an order of magnitude greater than what is currently available on the HST. This will allow one to study how the Galactic abundances of elements change with distance from the sun. As noted earlier, the ISM abundance values are more tightly constrained than stellar values, so this will help to better define Galactic abundance gradients.

\section{GALACTIC COSMIC RAYS}

Galactic cosmic rays (GCRs) constitute another sample of matter from beyond the solar system that provides information about the composition of matter in the Galaxy. Abundances of energetic nuclei near Earth reflect the source composition as well as the acceleration mechanism and transport processes that have affected these particles. Measurements of interstellar dust provide information related to the pool of material available for acceleration.

\section{Experimental technique}

Energetic ions in the cosmic rays have been measured with balloon- and space-based instruments for many years. Recent satellite detectors such as the Cosmic-Ray Isotope Spectrometer (CRIS) [56] on NASA's Advanced Composition Explorer (ACE) and the High Energy Telescope (HET) [57] on the joint ESA/NASA Ulysses spacecraft, as well as earlier instruments on ISEE-3 $[58,59,60]$, and Voyager 1 and $2[61,62,63]$ have provided GCR composition measurements for ions with energies less than $\sim 500 \mathrm{MeV} /$ nucleon. Typical spacecraft instruments use silicon detectors to measure the energy loss $(\mathrm{dE} / \mathrm{dx})$ and total energy for stopping nuclei. Trajectory information is provided by a scintillating opticalfiber hodoscope on ACE/CRIS, position-sensitive silicon wafers on Ulysses/HET, or gas-filled drift chambers on ISEE-3. At higher energies, the HEAO-3 Heavy Nuclei
Experiment (HNE) $[64,65]$ and the French-Danish C2 $[66,67]$ detectors used ionization chambers, Cherenkov counters and flash tubes to identify incident particles. Magnetic spectrometers have been flown as balloon payloads $[68,69,70]$.

In terms of galactic evolution, Galactic cosmic rays are a contemporary sample of matter; measurements of radioactive secondary isotopes such as ${ }^{10} \mathrm{Be}$ constrain the confinement time in the Galaxy to $\sim 15 \mathrm{Myr}$ using a simple leaky-box model [72]. Galactic cosmic rays are believed to sample sources in the local interstellar medium (ISM) within a few kiloparsecs of the solar system. Diffusion models of GCR propagation in the galactic disk and halo, constrained by diffuse $\gamma$-ray emission from cosmic-ray interactions with ISM protons [73], limit the dimension perpendicular to the disk of the volume sampled by GCRs [74, 73, 75]. If the diffusion is isotropic, particles undergoing a random walk are not likely to move farther along the disk than they can in a perpendicular direction into the halo. This means that the cosmic rays we observe sample a comparatively small volume around the solar system.

The composition of the source material from which galactic cosmic rays are accelerated is thought to be similar to an average of the ISM composition with possible additional contributions from stellar winds or supernova ejecta. A known elemental fractionation that depends on atomic parameters such as the first ionization potential (FIP) or condensation temperature (volatility) may reflect a fractionation of the source material or a preferential acceleration of dust grains [76]. Once accelerated, either by a single shock or by multiple encounters with shocks or turbulent magnetic fields, the particles face on average a $15 \mathrm{Myr}$ journey through a $\sim 5-10 \mathrm{~g} / \mathrm{cm}^{2}$ absorber, the interstellar medium. During this transport the relative abundances may be altered by spallation, radioactive decay, energy changing processes, and escape from the Galaxy. At the end of this the nuclei must still enter the heliosphere. The expanding solar wind, with its frozen-in magnetic field, tends to mix and lower the energies of the particles that are observed, modifying the spectra below a few $\mathrm{GeV} /$ nucleon.

Each of these processes must be modeled in order to understand the abundances at the source. Interstellar propagation calculations rely on measurements of the nuclear fragmentation cross sections, radioactive decay half-lives and the composition of the interstellar medium. For some species, secondary production by fragmentation overwhelms the primary contribution. The relative abundances of these isotopes tell us little about the source but do reflect the conditions during transport.

In spite of the many complex processes affecting the measured composition, galactic cosmic rays provide a rich variety of probes that constrain the parameters in the models. Elemental composition measurements have 
been made over a wide range in energy and mass. Now with ACE, we have a nearly complete set of precise isotopic GCR abundances from $\mathrm{He}$ to $\mathrm{Zn}$ below 500 $\mathrm{MeV} /$ nucleon. Data from HEAO-3, the Cosmic Ray Nucleus (CRN) experiment [71], and others at higher energies constrain the interstellar spectral indices which are essentially unaffected by heliospheric processes above $\sim 10 \mathrm{GeV} /$ nucleon.

Abundances for predominately secondary species depend on the details of transport through the Galaxy. Stable secondary isotopes constrain the total amount of matter the cosmic rays pass through. Radioactive $\beta$-decay secondaries probe the mean lifetime for escape from the Galaxy and the average density of the local interstellar medium [72]. Electron-capture secondaries reflect energy-changing processes in the interstellar medium and the possibility of multiple accelerations during propagation [77]. All of this information constrains the model predictions for the composition of the accelerated sample of material near the source.

Primary abundances allow the study of injection or acceleration fractionations imprinted on the source composition. Elements with similar FIP but different condensation temperatures can help distinguish between models of the GCR source. For example, low $\mathrm{Na} / \mathrm{Mg}, \mathrm{Ge} / \mathrm{Fe}$, $\mathrm{Cu} / \mathrm{Fe}$ ratios, or a high $\mathrm{P} / \mathrm{S}$ ratio in the GCR source compared to the solar-system abundances would favor models in which refractory cosmic rays are derived from ions sputtered from accelerated dust grains [78, 79]. Source ratios of these elements that are consistent with the solarsystem values might reflect a FIP-based fractionation such as that observed in stellar coronae. Radioactive primaries such as ${ }^{59} \mathrm{Ni}$ probe the history of the source material before acceleration. Stable refractory (or low-FIP) primaries offer a direct look at the mix of material in the source pool.

\section{Results}

Perhaps the most remarkable result from decades of cosmic-ray composition measurements is that the relative composition of the refractory isotopes is very similar to that of the solar system. It is somewhat surprising that this should be the case, given the 4.5 Gyr time difference between the formation of the solar system and the cosmic rays that we observe today. Figure 2 compares ACE measurements of GCR source abundances for iron-group cosmic rays to the corresponding solar system abundances, normalized separately to ${ }^{56} \mathrm{Fe}=1$ [80]. Further analysis indicates that the agreement also holds for ${ }^{54} \mathrm{Fe},{ }^{40} \mathrm{Ca}$, and for the extremely rare ${ }^{48} \mathrm{Ca}$ at $\sim 10^{-4}$ times the ${ }^{56} \mathrm{Fe}$ abundance [81]. The largest differences are less than a factor of two over nearly four orders of magnitude in absolute abundance. This similarity imposes limits on models of galactic chemical evolution over the time since the solar system was formed. It is reasonable to hypothesize that the mix of sources from which the cosmic rays are accelerated is very similar to that which contributed the material from which the solar system was formed.

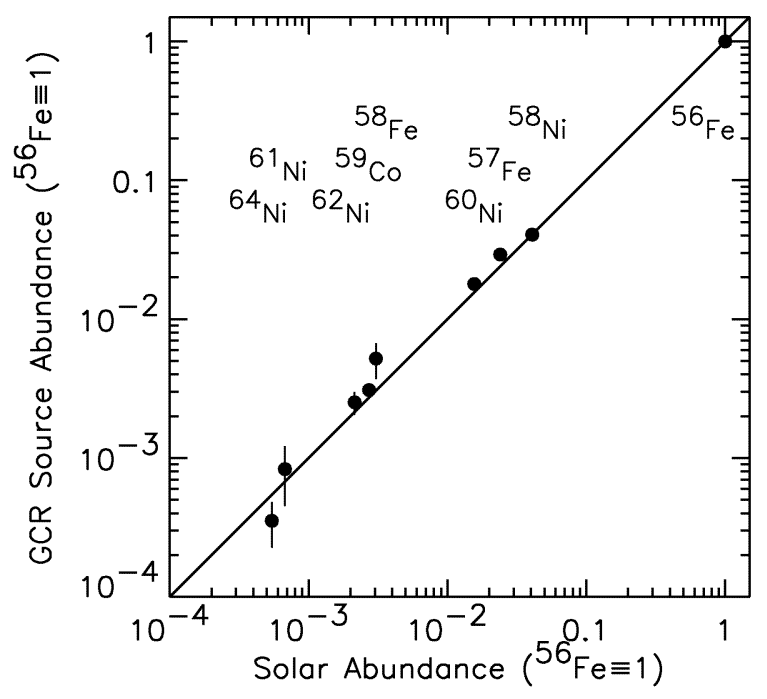

FIGURE 2. Comparison of cosmic-ray source abundances with corresponding solar-system values. Points along the diagonal line correspond to equal abundances, relative to ${ }^{56} \mathrm{Fe}$, in the two samples of material [taken from 80].

Differences in the relative composition of the GCRs and the solar system provide important insight into the nature of the source. A well-documented excess of ${ }^{22} \mathrm{Ne}$ in the cosmic-ray source [82] may be due to a small admixture of material from the surface layers of massive Wolf-Rayet stars which are enriched in the end products of core helium burning. If this is the case, one might expect enhancements of the neutron-rich isotopes of $\mathrm{Mg}$ and $\mathrm{Si}$, as well as heavy isotopes such as ${ }^{58} \mathrm{Fe},{ }^{59} \mathrm{Co}$, and ${ }^{61} \mathrm{Ni}$ [84]. A small excess of ${ }^{58} \mathrm{Fe}$ in the ACE data (see Figure 2) may fit a pattern of isotope enhancements predicted by the Wolf-Rayet models employed to explain the ${ }^{22} \mathrm{Ne}$ anomaly $[82,83]$.

Galactic cosmic ray abundances of ultra-heavy elements $(Z>30)$ show the imprints of stellar nucleosynthesis. The elements around $\mathrm{Z} \sim 39,57$, and 82 are dominated by contributions from isotopes produced in the (slow) "s-process" in which production chains stop at short-lived $\beta$-decay isotopes. Data from HEAO-3 HNE $[65,85]$ and Ariel-VI [86] show indications of enhancements for element groups which have contributions from nuclei formed predominately in the (rapid) "r-process" of explosive nucleosynthesis (e.g., in regions near $\mathrm{Z} \sim 34$, 
$53,65,77$, and the actinides). Production of these isotopes requires an environment in which neutron capture rates dominate over nucleon decay. Observation of a high ${ }^{78} \mathrm{Pt} /{ }^{82} \mathrm{~Pb}$ ratio by the TREK (track etch) experiment [87] also indicates an enhanced contribution of r-process material compared to the solar system. The actual site where the r-process occurs remains an unsolved mystery. It is clear, however, that both s- and r-process sources must contribute to account for the observed GCR abundances.

Primary isotopes such as ${ }^{59} \mathrm{Ni}$, which decay by electron capture, probe the time delay between cosmic-ray production and acceleration. ${ }^{59} \mathrm{Ni}$ is produced in supernovae and decays with a half-life of $\sim 10^{5}$ years. Once accelerated, the electrons are stripped off and the nuclei become essentially stable. Figure 3 shows the calculated abundance at Earth of ${ }^{59} \mathrm{Ni}$ and ${ }^{59} \mathrm{Co}$ as a function of the elapsed time before acceleration. The solid curves correspond to the fraction of mass-59 material originally produced as ${ }^{59} \mathrm{Ni}$. The ACE data (hatched area) is consistent with the complete decay of primary ${ }^{59} \mathrm{Ni}$ to ${ }^{59} \mathrm{Co}$, implying that a time longer than $10^{5}$ years elapsed before acceleration [88]. Since supernova shocks dissipate their energy over a $10^{4}$ year time-scale (assuming a typical ISM density), this rules out the possibility that the observed galactic cosmic rays come from supernovae accelerating their own ejecta.

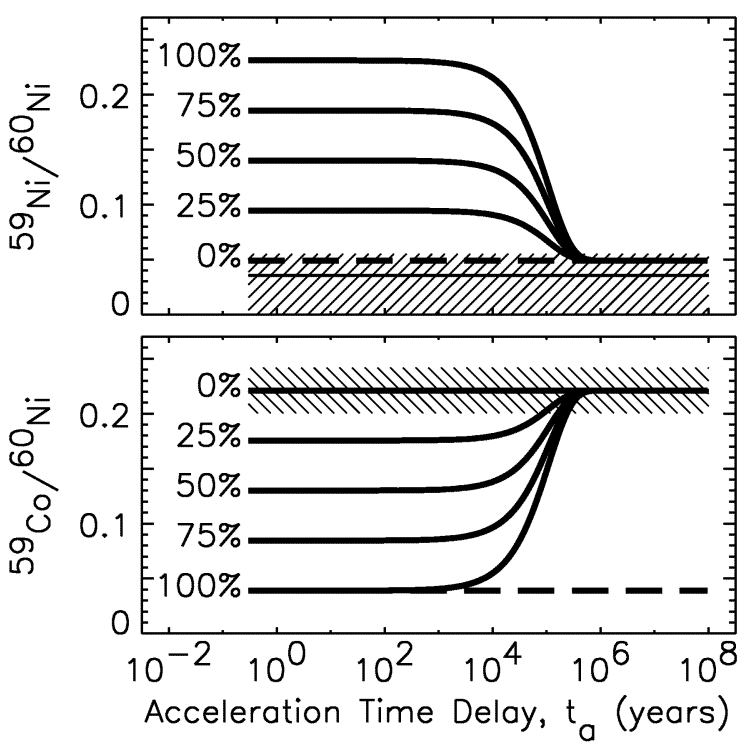

FIGURE 3. Abundances of mass- 59 isotopes relative to ${ }^{60} \mathrm{Ni}$ observed at Earth as a function of the time delay between production and acceleration. The solid curves correspond to the fraction of mass-59 isotopes originally produced as ${ }^{59} \mathrm{Ni}$ in the source. Hatched areas represent the region allowed by the ACE data, consistent with a time delay greater than $10^{5}$ years. [taken from 88]

The elemental fractionation inferred in the cosmic-ray source appears to be ordered by the first ionization po- tential or the generally correlated volatility. Determining which parameter controls the fractionation has strong implications for models of the source. A dependence on volatility would favor models involving a preferential acceleration of interstellar dust grains [76]. The source abundances of elements such as $\mathrm{Na}, \mathrm{P}, \mathrm{Cu}$, and $\mathrm{Ge}$ which break the general correlation of FIP and volatility tend to favor volatility models [78], as do the $\mathrm{Pb} / \mathrm{Pt}$-group results from the TREK detector exposed on Mir [87].

\section{Outstanding Problems}

The outstanding problems pertaining to galactic cosmic rays at this time relate to questions presented above. What is the nature of the cosmic-ray sources? Are there identifiable components of the source composition which differ from the average ISM? What exactly is the material that is being accelerated? Is the observed elemental fractionation a FIP or volatility effect? What is the acceleration history of the cosmic rays, do they see one or multiple accelerations?

\section{The Future}

Improvements in the propagation model input parameters will be important for significant future progress. Data from ACE for most isotopes have reached a statistical precision such that uncertainties in the calculated source abundances are dominated by the precision of the nuclear fragmentation cross sections, and radioactive decay parameters.

New measurements of the fragmentation cross sections from ${ }^{56} \mathrm{Fe}$ and ${ }^{60} \mathrm{Ni}$ on a hydrogen target were made in October 2000 at the GSI heavy ion synchrotron in Darmstadt, Germany. Analysis of these data is underway and the results should greatly improve the accuracy of propagation models in the iron-nickel region and for the sub-Fe isotopes down through $\mathrm{Ca}$ where $\mathrm{Fe}$ fragmentation is the dominant source of secondary production. These data will allow better constraints on the $\mathrm{Fe}-\mathrm{Ni}$ isotope excesses related to the acceleration time delay and Wolf-Rayet contributions. Future measurements of $\mathrm{Mg}, \mathrm{Si}$, and $\mathrm{S}$ fragmentation into $\mathrm{Na}$ and $\mathrm{P}$ would help distinguish between FIP and volatility while ultra-heavy production cross sections are needed to interpret the rprocess signatures.

New electron-attachment cross sections and radioactive decay parameters are also needed. The ACE data clearly show the effects of electron capture decay during transport [77] but cannot yet determine whether cosmic rays get their full energy from a single shock or are accelerated gradually in multiple shocks. The con- 
finement time in the Galaxy comes from analysis of radioactive decay secondaries such as ${ }^{10} \mathrm{Be},{ }^{26} \mathrm{Al},{ }^{36} \mathrm{Cl}$, and ${ }^{54} \mathrm{Mn}$ which sample different effective volumes corresponding to their decay half-lives. Decays of ${ }^{54} \mathrm{Mn}$ in the laboratory are dominated by capture of the attached electrons. In the cosmic rays, where the electrons have been stripped away, the much rarer $\beta^{-}$decay is much more important. A precise measurement of half-life for this branch would make it possible to determine whether $\mathrm{Fe}$ and lighter elements reside in the Galaxy for similar times.

The ACE data provide a rich set of measurements for isotopes up to $\mathrm{Zn}$. New measurements are needed for the ultra heavy species which include important signatures of $\mathrm{r}$ - and $\mathrm{s}$ - process nucleosynthesis as well as a number of important isotopes for distinguishing between FIP and volatility. The Advanced Cosmic-Ray Composition Experiment for the Space Station (ACCESS) has been proposed to measure elemental spectra up to Fe at energies approaching the "knee" $\left(\sim 3 \times 10^{15} \mathrm{eV}\right)$ to explore the limits of supernova shock acceleration. Abundances of elements with $\mathrm{Z}>30$ may also be measured, looking for enhancements of r-process elements and depletions of elements for which FIP and volatility are poorly correlated. The Heavy Nuclei Experiment (HNX) is a mission presently undergoing a Phase A study that would expose silicon detector stacks and glass track-etch detectors for three years. The HNX experiment would provide elemental composition measurements from zinc up through the actinides.

Another important proposed mission, the Interstellar Probe, would directly measure the energetic particle composition of the interstellar medium outside the heliosphere, free from the effects of solar modulation. This information is presently inferred from astronomical observations but would be an important constraint on propagation models. A currently operating mission, Voyager I, is thought to be nearing the boundary of the heliosphere and may have a chance to make composition measurements outside the termination shock within the next 10 15 years.

\section{Interstellar Dust}

Interstellar dust is a repository of the refractory elements in all but the warmest interstellar clouds and as such contains within it some of the keys toward our understanding of the history and evolution of the galaxy. Dust comprises roughly $1 \%$ of the mass of the interstellar medium, which in turn makes up a third of the mass of the galactic disk [89].

The composition of dust can be inferred from the depletion of atoms from the gas phase in the interstel- lar clouds [90] assuming a standard composition (gas + dust). The gas abundances are determined by spectroscopic observation of absorption lines along various lines of sight, as described in the previous section. The inferred dust phase contribution constrains dust models and serves as a cross check of the assumed composition. The standard abundances against which depletions are measured are generally the solar abundances but it is recognized that deviations from the standard are possible if the observed cloud is not well mixed [91].

The characteristic broadening of spectral lines in solids prevents precise determination of dust composition through astronomical means, however, considerable information can be obtained from extinction, polarization and emissivity measurements. Solid state transitions are observed to some degree as well as broadened molecular transitions. For example, the absence of the $\mathrm{OH}$ stretch at $3 \mu \mathrm{m}$ in absorption discounts the possibility of an ice-based composition of interstellar dust. Instead, the presence of this transition in cold dense interstellar clouds indicates that icy mantles have formed on the dust particles within them (see, e.g. discussion in [89]).

Fortunately the composition of local interstellar dust grains can be determined precisely though in situ measurements and sample return missions. The Ulysses and Galileo spacecraft have made direct measurements of the flux and size distribution of interstellar dust grains [92] within the solar system. However, grain sizes $<0.1 \mu \mathrm{m}$ are excluded from the inner heliosphere by radiation pressure and the interaction of the solar wind [93].

Future components of dust particle research include analysis of interstellar dust returned from the Stardust spacecraft. Although Stardust is primarily a comet sample return mission, some resources are devoted to interstellar dust collection. ESA is currently evaluating a proposed interstellar dust sample return mission (DUNE Galactic-Dust-Measurements-Near-Earth). Detailed analysis of smaller dust particles awaits a mission going outside the heliosphere such as the Interstellar Probe currently under study by NASA.

\section{STELLAR ABUNDANCES AND GALACTIC EVOLUTION}

Galactic abundances are neither constant in time and universal on a solar level [102] nor do they evolve in a simple fashion. Their evolution in space and time reflects the history of star formation and the lifetimes of the diverse contributing stellar objects like intermediate and low mass stars through planetary nebula ejection, mass loss from massive stars, as well as the ejecta of SNe II and SNe Ia. Planetary nebulae do contribute products of $\mathrm{H}$ - and He-burning and heavy s-process nuclei up to $\mathrm{Pb}$ 
and $\mathrm{Bi}$, based on a sequence of neutron captures and beta-decays during (shell) He-burning. Galactic evolution for the elements in the range $\mathrm{O}$ through $\mathrm{Ni}$ is dominated by two alternative explosive stellar sources, i.e by the combined action of SNe II and Ia. The site for the production of the heaviest elements up to $\mathrm{U}$ and $\mathrm{Th}$ (the r-process based on neutron captures in environments with very high neutron densities) is still debated and most probably related to supernovae and/or neutron star ejecta (from binary mergers or jets).

Stars (with understood exceptions) do not change the surface composition during their evolution. Thus, surface abundances reflect the interstellar medium (out of which the stars formed) at the time of their formation. Therefore, observations of the surface composition (via spectra) of very low metallicity stars (i.e. very old stars) give a clue to gas abundances throughout the evolution of our Galaxy. This way they can serve as a test for all the contributing stellar yields. Stellar abundances are measured as a function of metallicity $[\mathrm{Fe} / \mathrm{H}]=\log \left[(\mathrm{Fe} / \mathrm{H}) /(\mathrm{Fe} / \mathrm{H})_{\odot}\right]$. Typical (Galactic chemical) evolution calculations suggest roughly the "metallicity-age" relation $[\mathrm{Fe} / \mathrm{H}]=-1$ at $10^{9} \mathrm{y},-2$ at $10^{8} \mathrm{y}$, and -3 at $10^{7} \mathrm{y}$ (see e.g., $\left.[115,113,101]\right)$. Observational data for $[\mathrm{x} / \mathrm{Fe}]$ at low metallicities $(-2<[\mathrm{Fe} / \mathrm{H}]<-1)$, $\mathrm{x}$ standing for elements from $\mathrm{O}$ through $\mathrm{Ca}$, show an enhancement of the alpha elements $(\mathrm{O}$ through $\mathrm{Ca})$ by a factor of 2-3 (0.3 to $0.5 \mathrm{dex}$ in $[\mathrm{x} / \mathrm{Fe}])$ in comparison to Fe $[109,108,97,104,106,107,103,99,105,96]$. This is the clear fingerprint of the exclusive early contribution of fast evolving massive stars, i.e. SNe II. For $[\mathrm{x} / \mathrm{Fe}]>-$ 2 the scatter of this feature is very small. Below -2 it shows large variations of up to a factor of 10. As such very low metallicity stars were born when the Galaxy was only $10^{7}-10^{8} \mathrm{y}$ old, approaching at $\approx 10^{6} \mathrm{y}$ the lifetime of individual massive stars, it is clear that one does not obserse well mixed average features, but the results of individual stellar explosions.

There have been groundbreaking $\mathrm{r} / \mathrm{Fe}$ observations $[110,108,111,116,100,112]$ at low metallicities in recent years. The individual abundance features indicate a typical solar $\mathrm{r}$-abundance pattern, at least above Ba. A very important result is also that Th and $U$ could be observed. The r-abundances show a scatter of almost a factor of 1000 which still amounts to about a factor of 10 at $[\mathrm{Fe} / \mathrm{H}]=-1$, see Fig. 2 in [114]. This indicates on the one hand a large variation in $\mathrm{r} / \mathrm{Fe}$ ratios in the individual production sites, and on the other hand a rarer occurcance of r-process events as the asymptotic value is attained only later in galactic evolution. Nevertheless, the early occurrance of the r-process relates it to objects with massive stellar progenitors (either SNe II or neutron stars).

At about $-2<[\mathrm{Fe} / \mathrm{H}]<-1.5$ the onset of s-process nucleosynthesis from intermediate and low mass stars is observed, in agreement with the expected delay related to the stellar evolution timescales of such objects.

$\mathrm{SNe}$ Ia with their higher ratio of $\mathrm{Fe}$-group elements to $\mathrm{Si}-\mathrm{Ca}$ have to compensate for the respective overabundances in SNe II in order to obtain solar abundance ratios for the combined nucleosynthesis products. This effect is seen for metallicities in the range of $-1<[\mathrm{Fe} / \mathrm{H}]<0$. SNe II and $\mathrm{SNe}$ Ia, mixed in frequency ratios of $N_{\mathrm{Ia}} / N_{\mathrm{II}}=$ $0.15-0.27$ [98], require an $\mathrm{Fe}$ contribution from $\mathrm{SNe}$ Ia of $50-60 \%$, if average ejected Fe masses of $0.1 \mathrm{M}_{\odot}$ and $0.6 \mathrm{M}_{\odot}$ are taken for SN II and SN Ia. An interesting feature is that also the Fe-group ejecta of both types of supernovae have to differ. For $\mathrm{Ti}$ and $\mathrm{Mn}$ one finds at low metallicities average SN II values $[\mathrm{x} / \mathrm{Fe}]$ of 0.25 and -0.3 , i.e. a clear signatures for a SN II behavior different from solar, which asks for the opposite effect in SN Ia.

\section{ACKNOWLEDGEMENTS}

We thank the organizers of the SOHO/ACE workshop on "Solar and Galactic Composition" for their hospitality.

\section{REFERENCES}

1. Gloeckler, G., and Geiss, J., "Composition of the Local Interstellar Cloud from Observations of Interstellar Pickup Ions" in Solar and Galactic Composition, edited by R. F. Wimmer-Schweingruber, AIP Conf. Proc., Woodbury, NY, 2001, this volume.

2. Fisk, L. A., Kozlovsky, B., and Ramaty, R., Astrophys. J. Lett., 190, L35-L38 (1974).

3. Pesses, M. E., Jokipii, J. R., and Eichler, D., Astrophys. J. Lett., 246, L85-L89 (1981).

4. Hoppe, P., "Elemental and Isotopic Abundances in Meteorites", in Solar and Galactic Composition, edited by R. F. Wimmer-Schweingruber, AIP Conf. Proc., Woodbury, NY, 2001, this volume.

5. Thielemann, F.-K., Argast, D., Brachwitz, F., and Martinez-Pinedo, G., "Stellar Nucleosynthesis and galactic Abundances" in Solar and Galactic Composition, edited by R. F. Wimmer-Schweingruber, AIP Conf. Proc., Woodbury, NY, 2001, this volume.

6. Wurz, P., "Detection of Energetic Neutral Atoms", in The Outer Heliosphere: Beyond the Planets, Copernicus Gesellschaft, 2000, chap. 11, pp. 251-288.

7. Witte, M., Banaszkiewicz, M., and Rosenbauer, H., Space Sc. Rev., 78, 289-296 (1996).

8. Witte, M., Rosenbauer, H., Keppler, E., Fahr, H., Hemmerich, P., Lauche, H., Loidl, A., and Zwick, R., Astron. Astrophys. Suppl. Ser., 92, 333-348 (1992).

9. Witte, M., Rosenbauer, H., and Banaszkiewicz, M., $A d v$. Space Res., 13, 121-130 (1993).

10. Signer, P., Eberhardt, P., and Geiss, J., J. Geophys. Res., 70, 2243-2244 (1965).

11. Geiss, J., Eberhardt, P., Bühler, F., Meister, J., and Signer, P., J. Geophys. Res., 75, 5972 (1970).

12. Axford, W., Bühler, F., Eberhardt, P., and Geiss, J., J. Geophys. Res., 77, 6724 (1972). 
13. Bühler, F., Axford, W., Chivers, H., and Marti, K., J. Geophys. Res., 81, 111 (1976).

14. Lind, D., Geiss, J., and Stettler, W., J. Geophys. Res., 84, 6435 (1979).

15. Lind, D., Geiss, J., Bühler, F., and Eugster, O., "The Interstellar Gas Experiment, in: LDEF - 69 Months in Space”, in NASA Conf. Pub., 1991, vol. 3134, p. 585.

16. Bühler, F., Lind, D., Geiss, J., and Eugster, O., "The Interstellar Gas Experiment: Analysis in progress, in: LDEF 69 - Months in Space",in NASA Conf. Pub., 1993, vol. 3194, p. 705 .

17. Salerno, E., Bühler, F., Bochsler, P., Busemann, H., Eugster, O., Zastenker, N., Agafonov, Y., and Eismont, N., "Direct Measurement of ${ }^{3} \mathrm{He} /{ }^{4} \mathrm{He}$ in the LISM with the COLLISA Experiment" in Solar and Galactic Composition, edited by R. F. Wimmer-Schweingruber, AIP Conf. Proc., Woodbury, NY, 2001, this volume.

18. Brasken, M., and Kyrölä, E., Astron. Astrophys., 332, 732 (1998).

19. Keller, J., Ogilvie, K., and Coplan, M., "Measurement of Energetic Neutral Atom Composition in the Heliosphere and around Planetary Bodies" in Solar and Galactic Composition, edited by R. F. Wimmer-Schweingruber, AIP Conf. Proc., Woodbury, NY, 2001, this volume.

20. Stone, E. C., and Cummings, A. C., Proc. $27^{\text {th }}$ Intl. Cosmic Ray Conf. (Hamburg) 10, 4263-4266 (2001).

21. Klecker, B., Mewaldt, R. A., Bieber, J. W., Cummings, A. C., Drury, L., Giacalone, J., Jokipii, J. R., Jones, F. C., Krainev, M. B., Lee, M. A., Roux, J. A. L., Marsden, R. G., McDonald, F. B., McKibben, R. B., Steenberg, C. D., Baring, M. G., Ellison, D. C., Lanzerotti, L. J., Leske, R. A., Mazur, J. E., Moraal, H., Oetliker, M., Ptuskin, V. S., Selesnick, R. S., and Trattner, K. J., Space Sci. Rev., 83, 259-308 (1998).

22. Baker, D. N., Mason, G. M., Figueroa, O., Colon, G., Watzin, J. G. and Aleman, R. M., IEEE Trans. Geosci. \& Remote Sens., 31, 531-541 (1993).

23. Selesnick, R. S., Cummings, A. C., Cummings, J. R., Mewaldt, R. A., Stone, E. C., and von Rosenvinge, T. T., J. Geophys. Res., 100, (A6), 9503-9518 (1995).

24. Cummings, A. C., Stone, E. C., and Steenberg, C. D., Composition of Anomalous Cosmic Rays and Other Heliospheric Ions (2001), in preparation for submittal to JGR.

25. Gloeckler, G., Space Sci. Rev., 78, 335-346 (1996).

26. Slavin, J. D., and Frisch, P. C., The Ionization of Nearby Interstellar Gas, submitted to ApJ (2001).

27. Leske, R. A., Mewaldt, R. A., Cummings, A. C., Cummings, J. R., Stone, E. C., and von Rosenvinge, T. T., Space Sci. Rev., 78, 149-154 (1996).

28. Leske, R. A., Mewaldt, R. A., Christian, E. R., Cohen, C. M. S., Cummings, A. C., Slocum, P. L., Stone, E. C., von Rosenvinge, T. T., and Wiedenbeck, M. E., "Observations of Anomalous Cosmic Rays at 1 AU", in Acceleration and Transport of Energetic Particles Observed in the Heliosphere, edited by R. A. Mewaldt, J. R. Jokipii, M. A. Lee, E. Moebius, and T. H. Zurbuchen, AIP Conf. Proc., 528, Woodbury, 2000, pp. 293-300.

29. Leske, R. A., "Anomalous Cosmic Ray Composition from ACE", in 26th International Cosmic Ray Conference Invited, Rapporteur, and Highlight Papers, edited by B. L. Dingus, D. B. Kieda, and M. H. Salamon, AIP 516, Melville, 2000, pp. 274-282.
30. Klecker, B., McNab, M. C., Blake, J. B., Hamilton, D. C. Hovestadt, D., Kästle, H., Looper, M. D., Mason, G. M., Mazur, J. E., and Scholer, M., Astrophys. J. Lett., 442, L69-L72 (1995).

31. Mewaldt, R. A., Selesnick, R. S., Cummings, J. R., Stone, E. C., and von Rosenvinge, T. T., Astrophys. J. Lett., 466, L43-L46 (1996).

32. Barghouty, A. F., Jokipii, J. R., and Mewaldt, R. A., "The Transition from Singly to Multiply-Charged Anomalous Cosmic Rays: Simulation and Interpretation of SAMPEX Observations", in Acceleration and Transport of Energetic Particles Observed in the Heliosphere, edited by R. A. Mewaldt, J. R. Jokipii, M. A. Lee, E. Moebius, and T. H. Zurbuchen, AIP Conf. Proc., 528, Woodbury, NY, 2000, pp. 337-340.

33. Cummings, A. C., and Stone, E. C., Space Sci.Rev., 78, 117-128 (1996).

34. Sofia, U. J., Cardelli, J. A., and Savage, B. D., ApJ, 430, 650-666 (1994).

35. Snow, T. P., and Witt, A. N., Science, 270, 1455 (1995).

36. Snow, T. P., and Witt, A. N., ApJ, 468, L65-L68 (1996).

37. Mathis, J. S., ApJ, 472, 643-665 (1996).

38. Snow, T. P., Journal Geophys. Res., 105 A5, 10239-10248 (2000).

39. Sofia, U. J., and Meyer, D. M., ApJ, submitted (2001).

40. Jenkins, E. B., ApJ, 471, 292-301 (1996).

41. Savage, B. D., and Sembach, K. R., ApJ, 379, 245-259 (1991).

42. Joseph, C. L., and Jenkins, E. B., ApJ, 368, 201-214 (1991).

43. Sofia, U. J., "Limits to Galactic Abundances based on the Interstellar Medium", in Solar and Galactic Composition, edited by R. F. Wimmer-Schweingruber, AIP Conf. Proc., Woodbury, NY, 2001, this volume.

44. Jenkins, E. B., "Elemental Abuundances in the Interstellar Atomic Material", in Interstellar Processes, edited by D. J. Hallenbach and H. A. Thronson, Reidel, Dordrecht, 1987, pp. 533-559.

45. Savage, B. D., Cardelli, J. A., and Sofia, U. J., ApJ, 401, 706-723 (1992).

46. Savage, B. D., and Sembach, K. R., ARAA, 34, 229-330 (1996).

47. Cardelli, J. A., Meyer, D. M., Jura, M., and Savage, B. D., ApJ, 467, 334-340 (1996).

48. Sofia, U. J., Cardelli, J. A., Guerin, K. P., and Meyer, D. M., ApJ, 482, L105-L108 (1997).

49. Cardelli, J. A., and Meyer, D. M., ApJ, 477, L57-L60 (1997).

50. Meyer, D. M., Jura, M., and Cardelli, J. A., ApJ, 493, 222-229 (1998).

51. Sofia, U. J., Meyer, D. M., and Cardelli, J. A., ApJ, 522, L137-L140 (1999).

52. Federman, S. R., Lambert, D. L., Cardelli, J. A., and Sheffer, Y., Nature, 381, 764 (1996).

53. Lambert, D. L., Sheffer, Y., Federman, S. R., Cardelli, J. A., Sofia, U. J., and Knauth, D. C., ApJ, 496, 614-622 (1998).

54. Meyer, D. M., Cardelli, J. A., and Sofia, U. J., ApJ, 490, L103-L106 (1997).

55. Holweger, H., "Photospheric Abundances: Problems, Updates, Implications", in Solar and Galactic Composition, edited by R. F. Wimmer-Schweingruber, AIP Conf. Proc., Woodbury, NY, 2001, this volume. 
56. Stone, E.C., et al., Space Sci. Rev, 96, 285-356 (1998).

57. Simpson, J.A., et al., $A \& A S, 92,365$ (1992).

58. Greiner, D.E., et al., IEEE Trans. Geosci. Elec., GE-16, 163 (1978).

59. Wiedenbeck, M.E. and Greiner, D.E., ApJ, 247, L119L122 (1981).

60. Leske, R.A., Ap. J. 405, 567-583 (1993).

61. Stone, E.C., et al., Space Sci. Rev., 21, 355 (1977).

62. Lukasiak, A., et al., ApJ, 488, 454-461 (1997).

63. Webber, W.R., et al., ApJ, 457, 435-439 (1996).

64. Binns, W.R., et al., Nucl. Inst. Meth., 185, 415 (1981).

65. Binns, W.R., et al., ApJ 346, 997-1009 (1989).

66. Bouffard, M., et al., Astrophys. Space Sci., 84, 3 (1982).

67. Engelmann, J.J., et al., Astron. Astrophys., 233, 96-111 (1990).

68. Streitmatter, R.E., et al., Proc. $23^{\text {th }}$ Intl. Cosmic Ray Conf. (Calgary) 6, 623 (1993).

69. Moiseev, A., et al., ApJ, 474, 489 (1997).

70. Hams, T., et al., Proc. $26^{\text {th }}$ Intl. Cosmic Ray Conf. (Salt Lake City), 3, 121 (1999).

71. Grunsfeld, J.M. et al., ApJ, 327, L31-L34 (1988).

72. Yanasak, N.E., et al., ApJ, submitted (2001).

73. Bloemen, J.B.G.M., ARA\&A, 27, 469 (1989).

74. Stecker, F.W. and Jones, F.C., ApJ 217, 843 (1977).

75. Webber, W.R., Lee, M.A., and Gupta, M., ApJ 390, 96 (1992).

76. Meyer, J.P, Drury, L., and Ellison, D.C., ApJ 487, 182-196 (1997).

77. Niebur, S.M., et al., "Secondary Electron-Capture-Decay Isotopes and Implications for the Propagation of Galactic Cosmic Rays", in Acceleration and Transport of Energetic Particles Observed in the Heliosphere, AIP Conf. Proc., 528, Woodbury, NY, 2000, pp. 406-409.

78. George, J. S., Wiedenbeck, M. E., Barghouty, A. F., et al., "Cosmic Ray Source Abundances and the Acceleration of Cosmic Rays", in Acceleration and Transport of Energetic Particles Observed in the Heliosphere, edited by R. A. Mewaldt, J. R. Jokipii, M. A. Lee, E. Moebius, and T. H. Zurbuchen, AIP Conf. Proc., 528, Woodbury, NY, 2000, pp. 437-440.

79. George, J. S., Wiedenbeck, M. E., Binns, W. R., et al., "The Phosphorus/Sulfur Abundance Ratio as a Test of Galactic Cosmic-Ray Source Models", in Solar and Galactic Composition, edited by R. F. WimmerSchweingruber, AIP Conf. Proc., Woodbury, NY, 2001, this volume.

80. Wiedenbeck, M. E., et al., "Constraints on Cosmic-Ray Acceleration and Transport from Isotope Observations", in Acceleration and Transport of Energetic Particles Observed in the Heliosphere, edited by R. A. Mewaldt, J. R. Jokipii, M. A. Lee, E. Moebius, and T. H. Zurbuchen, AIP Conf. Proc, 528, Woodbury, NY, 2000,pp. 363-370.

81. Wiedenbeck, M. E., et al., Space Sci. Rev., accepted (2001).

82. Binns, W. R., Wiedenbeck, M. E., et al., "Galactic Cosmic Ray Neon Isotope Abundances Measured on ACE", in Acceleration and Transport of Energetic Particles Observed in the Heliosphere, edited by R. A. Mewaldt, J. R. Jokipii, M. A. Lee, E. Moebius, and T. H. Zurbuchen, AIP Conf. Proc., 528, Woodbury, NY, 2000, pp. 413-416.

83. Binns, W. R., Wiedenbeck, M. E, Christian, E. R., et al., "GCR Neon Isotopic Abundances: Composition with Wolf-Rayet Star Models and Meteoritic Abundances", in Solar and Galactic Composition, edited by R. F. Wimmer-Schweingruber, AIP Conf. Proc., Woodbury, NY, 2001, this volume.

84. Prantzos, N. et al., Proc. $19^{\text {th }}$ Intl. Cosmic Ray Conf. (La Jolla) 3, 167-170 (1985).

85. Binns, W. R., et al., "The Abundances of the Heavier Elements in the Cosmic Radiation", in Cosmic Abundances of Matter, (ed. C.J. Waddington), AIP Conf. Proc., 183, 147 (1989).

86. Fowler, P. H., et al., ApJ, 314, 739 (1987).

87. Westphal, A. J., et al., Nature, 357, 50 (1988).

88. Wiedenbeck, M. E., et al., ApJ, 523, L61-L64 (1999).

89. Whittet, D. C. B., Dust in the Galactic Environment, Institute of Physics Publishing, New York (1992).

90. Sofia, U.J., ASP Conf. Series, 122, 77 (1997).

91. Geis, D. R. and Lambert, D. L., ApJ, 427, 232 (1992).

92. Frisch, P. C., et al., ApJ, 525, 492 (1999).

93. Linde, T. J. and Gombosi, T. I., J. Geophys. Res., 105 10411-10417 (2000).

94. DuVernois, M. A. and Thayer, M. R., ApJ, 465, 982-984 (1996).

95. Ormes, J.F. and Frier, P. ApJ, 222, 471 (1978).

96. Argast, D., Samland, M., Gerhard, O.E., and Thielemann, F.-K. , $A \& A$, 356, 873 (2000).

97. Boesgaard A.M., King J.R., Deliyannis C.P., and Vogt S.S., ApJ, 117, 492 (1999).

98. Cappellaro, E. et al. $A \& A$, 322, 431 (1997).

99. Carretta, E., Gratton, R.G., and Sneden, C., $A \& A, \mathbf{3 5 6}$, 238 (2000).

100. Cayrel, R. et al., Nature, 409, 691 (2001).

101. Chiappini, C., Matteucci, F., Beers, T. C., Nomoto, K., ApJ, 515, 226 (1999).

102. Grevesse, N. and Sauval, A. J., Space Sci. Rev., 85, 161 (1998).

103. Idiart T. and Thévenin F., ApJ, 541, 207 (2000).

104. Israelian G., García López R.J., and Rebolo R., , ApJ, 507, 805 (1998).

105. Israelian G., Rebolo R., García López R.J., et al., ApJ, 551, 833 (2001).

106. Jehin E., Magain P., Neuforge C., et al., $A \& A, 341,241$ (1999).

107. Matteucci, F., Romano, D., and Molaro, P., $A \& A$, 341, 458 (1999).

108. McWilliam, A., Ann. Rev. Astron. Astrophys., 35, 503, (1997).

109. Primas F., Molaro P., and Castelli F., $A \& A, \mathbf{2 9 0}, 885$ (1994).

110. Sneden, C., et al., ApJ, 467, 819-840 (1996).

111. Sneden, C., et al., ApJ, 533, L139-L142 (2000).

112. Sneden, C., et al., ApJ, 536, L85-L88 (2000).

113. Thomas, D., Greggio, L., and Bender, R., MNRAS, 296, 119, (1998).

114. Truran, J.W., Cowan, J.J., Sneden, C., Burris, D.L., and Pilacowski, C.A., in The First Stars, Springer, eds. A. Weiss et al. (2000).

115. Tsujimoto, T., et al. , MNRAS, 277, 945 (1995).

116. Westin J., et al., ApJ, 530, 783 (2000). 